\title{
A false-positive finding in therapentic evaluation: hypermetabolic axillary lymph node in a lymphoma patient following FDG extravasation
}

Thomas Wagner, Nicolas Brucher, Anne Julian, Anne Hitzel Toulouse Purpan University Hospital Nuclear Medicine Department

[Received 21 V 2011; Accepted 16 XI 2011]

\begin{abstract}
We report a case of a false-positive finding in FDG PET/CT following radiotracer extravasation. A 15-year-old male patient was referred for therapeutic evaluation status post-chemotherapy for a lymphoblastic lymphoma. FDG PET/CT showed discordant findings with a marked decrease in a liver/hepatic hilum uptake, disappearance of a subcutaneous left supraclavicular uptake, and appearance of intense right axillary nodal uptake. Extravasation in the right superior limb was noted. Comparison with the previous scan showed that the axillary nodes were present, measured less than $1 \mathrm{~cm}$ in their short axis, had not increased in size, and had a fatty hilum. We concluded that FDG uptake was caused by a migration in lymphatic vessels.
\end{abstract}

Key words: FDG PET/CT, therapy response assessment, extravasation

Nuclear Med Rev 2011; 14, 2: 109-111

Correspondence to: Dr. Thomas Wagner

Nuclear Medicine Department

Purpan University Hospital

Place du Docteur Baylac

TSA 40031, 31059 Toulouse cedex 9, France

Tel: + 33561772129 , fax: + 33561777592

e-mail: thomwagner@hotmail.com

\section{Introduction}

FDG PET/CT is increasingly being used and investigated for therapy response in a wide variety of cancers, e.g. lung cancer [1], rectal cancer [2], oesophageal cancer [3], and lymphoma [4] and in a larger number of indications: neoadjuvant chemotherapy in breast cancer [5], radiochemotherapy in rectal cancer [6], and early response to chemotherapy in lymphoma [7]. Hodgkin's disease and non-Hodgkin lymphoma are two malignancies where FDG PET has become central for appropriate patient management. The accuracy of therapy response assessment is therefore paramount and nuclear medicine physicians must be aware of all pitfalls and causes of false-positive and false-negative findings with FDG PET/CT. We describe a false-positive finding in a young male patient following radiotracer extravasation.

\section{Case report}

A 15-year-old male patient was referred to our institute for therapeutic evaluation status post-chemotherapy for a lymphoblastic lymphoma. FDG PET/CT was compared to the pretherapeutic scan. Discordant findings were noted: there was a marked decrease in a previous liver/hepatic hilum uptake, disappearance of a subcutaneous left supraclavicular uptake, and appearance of intense right axillary nodal uptake (Figure 1). There was a large area of radiotracer uptake within subcutaneous tissue of the right superior limb, consistent with extravasation (Figure 2). The axillary nodes were present on the previous scan, were not hypermetabolic, measured less than $1 \mathrm{~cm}$ in their short axis, had not increased in size, and had a fatty hilum (Figure 3). The discordant findings led us to hypothesize that the right axillary lymph node uptake was due to radiotracer extravasation to the 

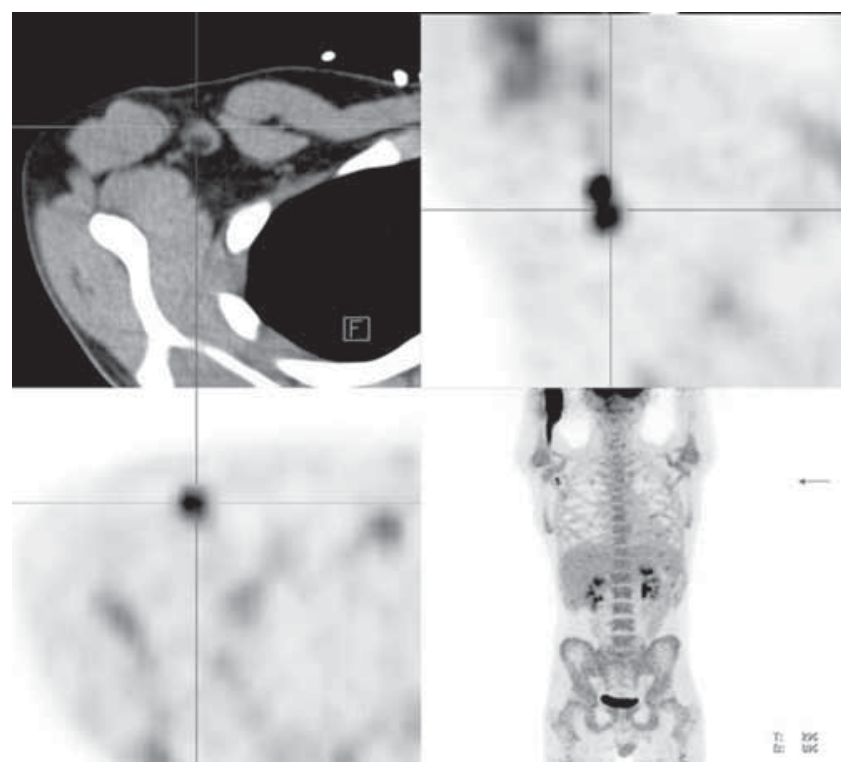

Figure 1. Intense FDG uptake within a right axillary lymph node. The lymph node has a fatty hilum and measures less than $1 \mathrm{~cm}$ in its short axis. MIP images show radiotracer extravasation within the right superior limb and intense FDG uptake within the right axillary fossa. Top left: axial CT section; top right: coronal PET section; bottom left: axial PET section; bottom right: MIP.
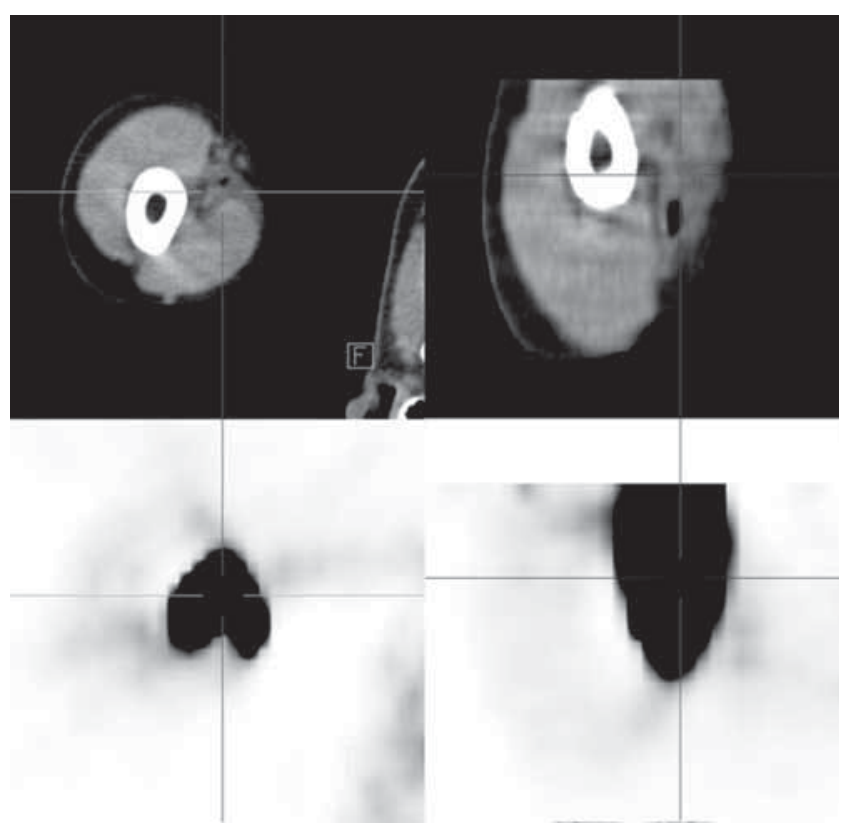

Figure 2. Intense FDG accumulation within subcutaneous tissue of the right superior limb caused by radiotracer extravasation. Top left: axial CT section; bottom left: axial PET section; top right: coronal CT section; bottom right: coronal PET section.

subcutaneous tissue and radiotracer incorporation within lymphatic vessels and lymph nodes. We concluded that the axillary lymph node uptake was a false-positive and that the patient was in partial metabolic response.

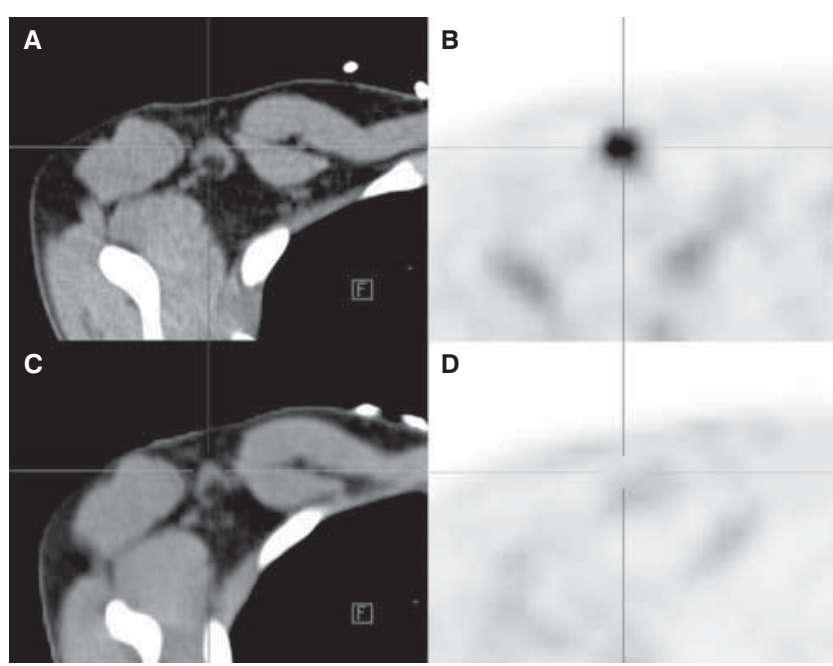

Figure 3. Comparison with the previous FDG PET/CT of two months earlier. Appearance of intense FDG uptake within right axillary lymph nodes. Comparison on the CT does not show an increase in size, nodes present with a fatty hilum and are subcentimetric in their short axis.

A. Axial interim CT; B. Axial interim PET; C. Axial pre-therapeutic CT; D. Axial pre-therapeutic PET.

\section{Discussion}

Therapy response assessment is a critical step in patient management and has become an increasingly important part of our daily clinical workload at PET/CT centres. Discordant findings are some of the most difficult situations to be confronted with. A deep understanding of pathologies and of therapeutics alongside extensive technical skills has become indispensable for nuclear medicine physicians, and will help when reporting on complex scans. The case report we describe here is an example of a complex scan where discordant findings are to be interpreted in the context of an overall decrease or disappearance of previous FDG uptake sites and where the appearance of an FDG avid lymph node is situated next to an area of radiotracer extravasation. FDG accumulation within the axilla following subcutaneous radiotracer infiltration has been described before with FDG [8] and with bone tracer MDP [9] We feel that it is important for nuclear medicine physicians to be aware of this cause of false-positive findings, especially in a time where patient management in oncology depends increasingly on FDG PET/CT results.

\section{References}

1. Hicks RJ. Role of 18F-FDG PET in assessment of response in non-small cell lung cancer. J Nucl Med 2009; 50: 31S-42S.

2. Capirci C, Rampin L, Erba PA et al. Sequential FDG-PET/CT reliably predicts response of locally advanced rectal cancer to neo-adjuvant chemo-radiation therapy. Eur J Nucl Med Mol Imaging 2007; 34: 1583-1593.

3. Sendler A. Metabolic response evaluation by PET during neoadjuvant treatment for adenocarcinoma of the esophagus and esophagogastric junction. Recent Results Cancer Res 2010; 182: 167-177. 
4. Hutchings M, Barrington SF. PET/CT for therapy response assessment in lymphoma. J Nucl Med 2009; 50: 21S-30S.

5. Groheux D, Giacchetti S, Espie M, Rubello D, Moretti JL, Hindie E. Early monitoring of response to neoadjuvant chemotherapy in breast cancer with (18)F-FDG PET/CT: defining a clinical aim. Eur J Nucl Med Mol Imaging 2011; 38: 419-425.

6. Janssen $\mathrm{MH}$, Ollers MC, van Stiphout RG et al. Evaluation of early metabolic responses in rectal cancer during combined radiochemotherapy or radiotherapy alone: sequential FDG-PET-CT findings. Radiother Oncol 2010; 94: 151-155.
7. MacManus MP, Seymour JF, Hicks RJ. Overview of early response assessment in Iymphoma with FDG-PET. Cancer Imaging 2007; 7 : 10-18.

8. Chiang SB, Rebenstock A, Guan L, Burns J, Alavi A, Zhuang H. Potential false-positive FDG PET imaging caused by subcutaneous radiotracer infiltration. Clin Nucl Med 2003; 28: 786-788.

9. Dogan AS, Rezai K. Incidental lymph node visualization on bone scan due to subcutaneous infiltration of Tc-99m MDP. A potential for false positive interpretation. Clin Nucl Med 1993; 18: 208-209. 